Document downloaded from:

http://hdl.handle.net/10251/94491

This paper must be cited as:

De Rosario Martínez, H.; Belda Lois, JM.; Fos Ros, F.; Medina Ripoll, E.; Poveda Puente, R.; Kroll, M. (2014). Correction of joint angles from kinect for balance exercising and assessment. Journal of Applied Biomechanics. 30(2):294-299. doi:10.1123/jab.2013-0062

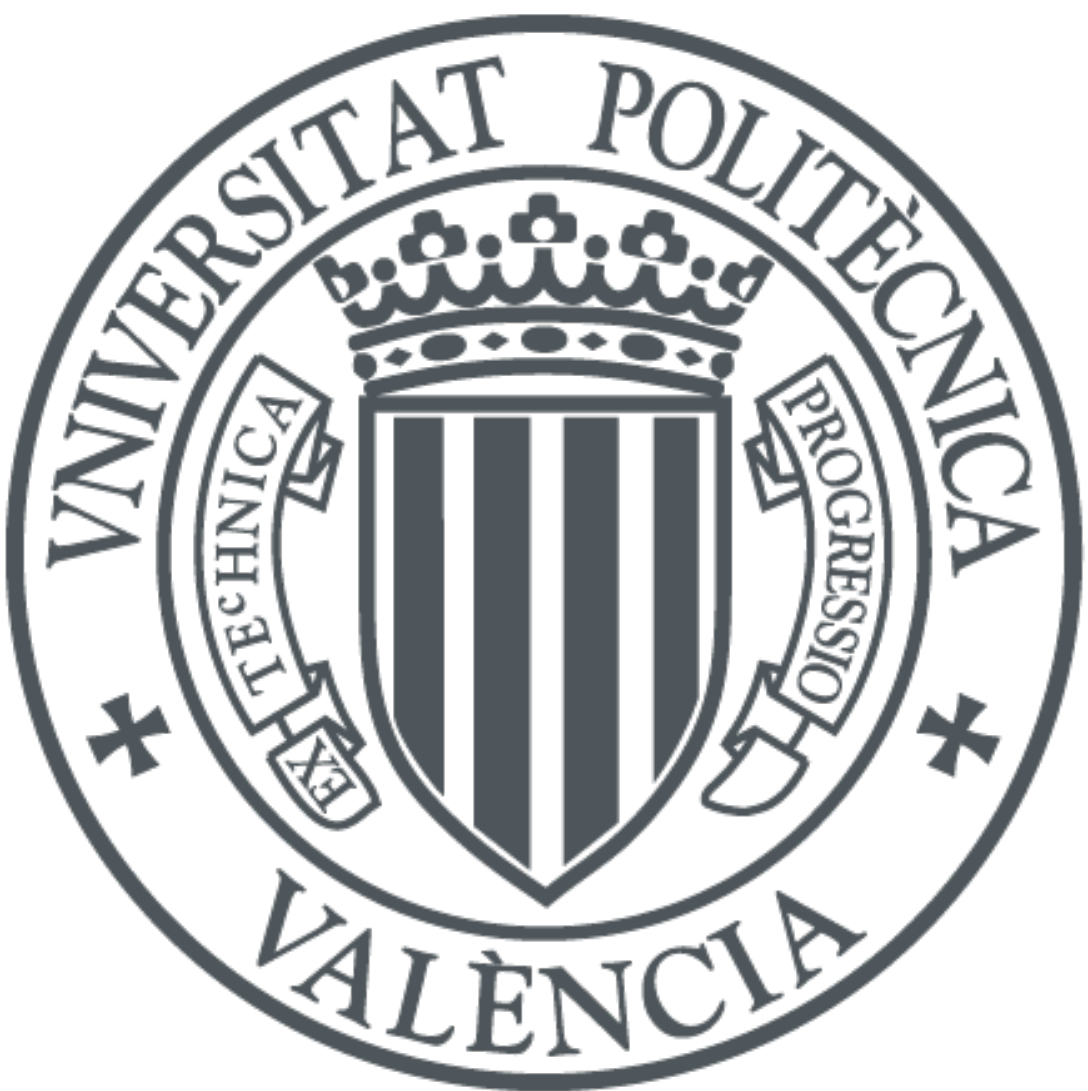

The final publication is available at

http://doi.org/10.1123/jab.2013-0062

Copyright Human Kinetics

Additional Information 


\title{
Correction of joint angles from Kinect for balance exercising and assessment
}

Helios De Rosario, ${ }^{1,2}$ Juan Manuel Belda-Lois, ${ }^{1,2}$ Francisco Fos, ${ }^{1}$ Enrique Medina, ${ }^{1}$ Rakel Poveda-Puente, ${ }^{1}$ Michael Kroll ${ }^{3}$

\author{
${ }^{1}$ Instituto de Biomecánica de Valencia, Valencia, Spain \\ ${ }^{2}$ CIBER de Bioingeniería, Biomateriales y Nanomedicina (CIBER-BBN), Spain \\ ${ }^{3}$ Deutsche Sporthochschule Köln, Cologne, Germany
}

Correspondence address: Helios De Rosario. Instituto de Biomecánica de Valencia, Universitat Politècnica de Valencia, Edificio 9C, Camino de Vera s/n, E-46022, Valencia, Spain. Tel: +34 963879160. Fax: +34963879169. E-mail: helios.derosario@ibv.upv.es

\begin{abstract}
The new generation of videogame interfaces such as Microsoft's Kinect opens the possibility of implementing exercise programs for physical training, and of evaluating and reducing the risks of elderly people falling. However, applications such as these might require measurements of joint kinematics that are more robust and accurate than the standard output given by the available middleware. This paper presents a method based on particle filters for calculating joint angles from the positions of the anatomical points detected by PrimeSense's NITE software. The application of this method to the measurement of lower limb kinematics reduced the error by one order of magnitude, to less than $10^{\circ}$, except for hip axial rotation, and it was advantageous over inverse kinematic analysis, in ensuring a robust and smooth solution without singularities, when the limbs are out-stretched and anatomical landmarks are aligned.
\end{abstract}

Keywords: particle filter, human movement analysis, video games 


\section{Introduction}

The benefit of "exergames" in stimulating sensorimotor learning processes ${ }^{1}$ has encouraged the creation of therapy programs based on new-generation videogames, using motion capture devices that reduce the distance between biomechanical research, clinical practice, and home-based interventions.

Besides Nintendo's Wii, ${ }^{2-4}$ Microsoft's Kinect is a popular videogame platform used for balance-recovery programs. ${ }^{5-7}$ In addition to motivating and guiding the user, Kinect can also be used to monitor the exercises for assessment and feedback purposes. A successful application, however, requires a trade-off between the amount and quality of motion data, and the accuracy that the sensors can provide. Many studies have used the OpenNI interface to the Kinect sensor, and PrimeSense's NITE toolbox. ${ }^{8}$ This solution yields position errors of 1-to-10 cm, ${ }^{9,10}$ which can be acceptable for the proposed applications. Microsoft's Software Development Kit, which uses "randomized decision forests," errors of joint rotations, which are also given by modern versions of those interfaces. The only published study that we have found reported mean errors of less than $10^{\circ} ;^{15}$ however, NITE's documentation warns about the important noise of joint orientations, and their indetermination when limb segments are aligned. ${ }^{8}$

This paper investigates an alternative resolution of the inverse kinematic problem for the "iStoppFalls" balance-training and assessment program, ${ }^{16}$ using particle filters (PF). This technique is normally used to analyze complex images, formed by point clouds and volumes. The present study will explore its application to the analysis of higher-level, simpler positional data provided by NITE, and test whether it can improve the angle estimations given by that middleware.

\section{Materials and methods}

\section{Skeleton and PF model}

Since the program of exercises focused on the motion of limbs, the model took the trunk as the root segment, from which the arms and legs stemmed as two-segment linkages (Figure 1, left). Three rotational degrees of freedom were assigned to trunk, shoulders and hips, and one to elbows and knees. 

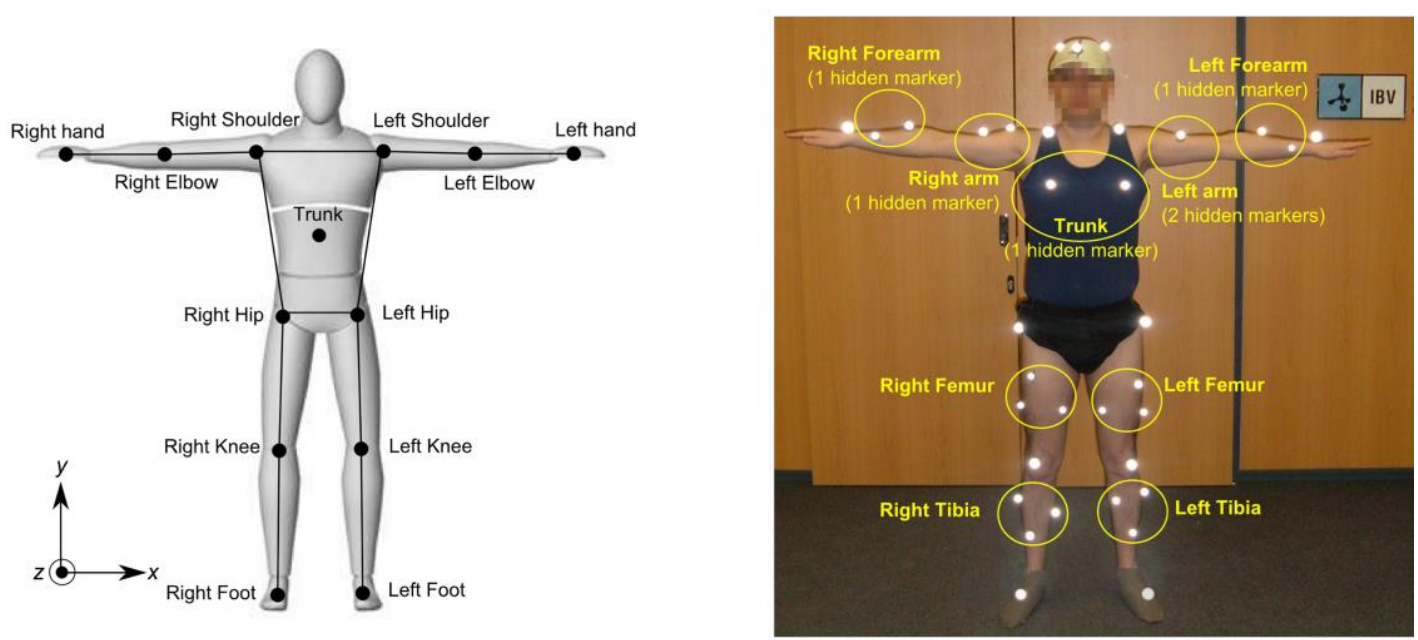

Figure 1 - Left: skeleton model in the reference posture (null rotation of all joints). Right: set of markers used for motion capture with marker sets of body segments highlighted.

Trunk displacement was irrelevant for the purposes of the study. Thus, the 3D positions of the remaining 12 joints were represented by their distance to the trunk joint, with their 36 coordinates gathered in vector $\mathbf{y}_{t}$ for each instant $t$. Human joint rotations are usually represented as Euler sequences, ${ }^{17,18}$ but for computational purposes, we used vector $\boldsymbol{\theta}_{t}$, which contained the 19 coordinates of the joint relative attitude vectors. ${ }^{19}$ Given the body segments' length, the relation between "error-free" measurements of $\mathbf{y}_{t}$ and $\boldsymbol{\theta}_{t}$ was determined by the direct kinematic model:

$$
\mathbf{y}_{t}=\mathcal{K}\left(\boldsymbol{\theta}_{t}\right)
$$

A PF was used to calculate $\boldsymbol{\theta}_{t}$ from $\mathbf{y}_{t}$. Both vectors were considered random variables, whose dynamic behavior was modeled by a Markovian Stochastic Process:

$$
\begin{gathered}
\boldsymbol{\theta}_{t} \sim f\left(\boldsymbol{\theta} \mid \boldsymbol{\theta}_{t-1}\right), \\
\mathbf{y}_{t} \sim g\left(\mathbf{y} \mid \boldsymbol{\theta}_{t}\right) .
\end{gathered}
$$

The values of $\boldsymbol{\theta}_{\boldsymbol{t}}$ were obtained recursively as the average of 512 particles $\left\{{ }^{i} \boldsymbol{\theta}_{t}\right\}_{i=1}^{512}$, which were propagated by the "sample-resample" technique, as follows. An initial population of particles with fixed values $\left\{{ }^{i} \boldsymbol{\theta}_{0}\right\}$ was first defined. In each later instant $t$, the a priori distribution of $\boldsymbol{\theta}_{t}$ was estimated by sampling the values $\left\{i \tilde{\boldsymbol{\theta}}_{t}\right\}$ out of (2), given $\left\{{ }^{i} \boldsymbol{\theta}_{t-1}\right\}$. Then, the a posteriori distribution was estimated by assigning weights 
to $\left\{\tilde{\boldsymbol{\theta}}_{t}\right\}$ after (3) and the observed value of $\mathbf{y}_{t}$. Then $\left\{{ }^{i} \boldsymbol{\theta}_{t}\right\}$ were resampled with replacement from $\left\{{ }_{i} \tilde{\boldsymbol{\theta}}_{t}\right\}$, according to those weights. This algorithm ensures that particles remain in regions of high probability, avoids accumulation of errors over time, and gives stable results. ${ }^{20}$

\section{Model training}

For the sake of simplicity and computational efficiency, the functions defined in (2) and (3) were based on multivariate normal distributions. Their means were defined on theoretical grounds, such that $f\left(\boldsymbol{\theta} \mid \boldsymbol{\theta}_{t-1}\right)$ described a random walk, and $g\left(\mathbf{y} \mid \boldsymbol{\theta}_{t}\right)$ was centered around the value of the direct kinematic model:

$$
\begin{gathered}
E\left(f\left(\boldsymbol{\theta} \mid \boldsymbol{\theta}_{t-1}\right)\right)=\boldsymbol{\theta}_{t-1}, \\
E\left(g\left(\mathbf{y} \mid \boldsymbol{\theta}_{t}\right)\right)=\mathcal{K}\left(\boldsymbol{\theta}_{t}\right) .
\end{gathered}
$$

Limb lengths, required by $\mathcal{K}(\boldsymbol{\theta})$, were obtained by an initial calibration, through measuring distances between joints in the first static instants. $f\left(\boldsymbol{\theta} \mid \boldsymbol{\theta}_{t-1}\right)$ was trimmed to retain the possible values of $\boldsymbol{\theta}_{t}$ within the ranges of motion of healthy adults. $^{21,22}$

The covariance matrices of both distributions $\left(\boldsymbol{\Sigma}_{\theta}, \boldsymbol{\Sigma}_{y}\right)$ were obtained experimentally with a volunteer. The work was approved by the ethical committee of the Universitat Politècnica de València, and the subject gave informed consent to participate.

Two 10-second static measurements were taken with Kinect, in order to estimate the covariance of $g\left(\mathbf{y} \mid \boldsymbol{\theta}_{t}\right)$. Medium-to-high correlations were observed between the variations of some coordinates of $\mathbf{y}_{t}$; therefore, a full matrix was defined for $\boldsymbol{\Sigma}_{y}$.

Then, cyclic rotations around the different axes of each joint, starting in the " $\mathrm{T}$ posture," were recorded separately in 10-second measurements, using the Kinescan-IBV photogrammetry system with 10 high-resolution cameras, and 42 markers that facilitated the calculation of joint positions from a well-defined kinematic model (Figure 1, right). ${ }^{23}$ Thus, the size of the difference $\boldsymbol{\theta}_{t}-\boldsymbol{\theta}_{t-1}$ was considered to model the variability of $f\left(\boldsymbol{\theta} \mid \boldsymbol{\theta}_{t-1}\right)$. $\boldsymbol{\Sigma}_{\theta}$ was assumed diagonal. 
The stochastic behavior of the model was assumed symmetric. This property was forced by averaging the variances of the left and right joints for both covariance matrices.

\section{Comparison of joint rotations}

The joint rotation errors of the NITE and PF algorithms were compared for the main movements that must be done by the user during the "iStoppFalls" exercises: "back knee" (alternate knee flexion while standing), "sit+front knee" (sit and alternate leg elevation), "side hip" (alternate lateral leg elevation while standing), and "step", based on the Otago strengthening program, ${ }^{24}$ plus "near tandem" (keep standing with one foot in front of the other) from the "Quick-Screen" fall risk assessment. ${ }^{25}$

These exercises were done by the volunteer and recorded simultaneously by Kinect and Kinescan-IBV. In each exercise, the subject started in the "T-posture," to calibrate limb lengths and set the initial value of $\boldsymbol{\theta}_{0}$. In all cases, the exercise was done with the right-hand side of the body, in order to evaluate whether the results varied depending on the level of motion.

Overall, there were three sets of results: (1) the "gold standard" of KinescanIBV, (2) the values obtained by the NITE algorithm from Kinect data, and (3) the results of the PF. The output of NITE, given as rotation matrices, was transformed into attitude vectors for comparison. As data analysis revealed frequent axial "flips" of the limbs, as if an axial rotation of $180^{\circ}$ along their long axes had been added, an opposite rotation was introduced at such discontinuities, in order to avoid error inflation.

The errors of the two Kinect-based results were modeled as motion artifacts added to the "correct" movement represented by the gold standard. ${ }^{26}$ This resulted in the "error attitude vectors" ${ }^{A} \boldsymbol{\varepsilon}_{t}^{j}$, with different values for each joint $j$ and for each algorithm A. The analysis was focused on hips and knees, the joints of interest for the target application.

These errors were represented as Euler angles for the standard sequence of lower limbs (flexion-abduction-axial rotation). For a more concise comparison of the total amount of error in each joint, the difference between the modules of ${ }^{A} \boldsymbol{\varepsilon}_{t}^{j}$ was also calculated at each instant:

$$
\Delta \varepsilon_{t}^{j}=\left|{ }^{N I T E} \boldsymbol{\varepsilon}_{t}^{j}\right|-\left|{ }^{P F} \boldsymbol{\varepsilon}_{t}^{j}\right|
$$


These variables were a measure of the "improvement" provided by the PF. To verify that such an improvement was significant, a Wilcoxon signed-rank test was applied to the distributions of $\Delta \boldsymbol{\varepsilon}_{t}^{j}$. Their values were also calculated with a four-times smaller or larger set of particles (128 or 2048), in order to evaluate the potential impact of changing the ratio between the variability of the model and the number of particles. All the calculations were done with GNU Octave. ${ }^{27}$

\section{Results}

Standard deviations of $f\left(\boldsymbol{\theta} \mid \boldsymbol{\theta}_{t-1}\right)$ and $g\left(\mathbf{y} \mid \boldsymbol{\theta}_{t}\right)$ were between $1^{\circ}$ and $6^{\circ}$, and between 6 and $40 \mathrm{~mm}$, respectively, with the remarkable exception of the error in the position of the hand, which was over $150 \mathrm{~mm}$ in the camera plane (Table 1).

The absolute values of the Euler angle errors had a great dispersion. Their distributions were mainly concentrated between $1^{\circ}$ and $10^{\circ}$, but NITE errors were one order of magnitude greater (tens of degrees) for hip abduction and for knee flexion of both sides (Figure 2). When all the measures were taken together, the NITE average error for right hip flexion was also greater than the corresponding PF error, although both distributions had the same order of magnitude (Table 2). On the other hand, the PF did not improve the error of NITE in hip axial rotation, and in fact, it was substantially increased for the left-hand side.

Table 1. Standard deviations (SD) used to model the distributions of the particle filter.

\begin{tabular}{|c|c|c|c|c|c|c|}
\hline & \multicolumn{3}{|c|}{$\mathrm{SD}\left(f\left(\boldsymbol{\theta} \mid \boldsymbol{\theta}_{t}\right)\right)\left(^{\circ}\right)$} & \multicolumn{3}{|c|}{$\mathrm{SD}\left(g\left(\mathbf{y} \mid \boldsymbol{\theta}_{t}\right)\right)(\mathrm{mm})$} \\
\hline & $x$ & $y$ & $z$ & $x$ & $y$ & $z$ \\
\hline Trunk & 1.60 & 2.44 & 1.16 & - & - & - \\
\hline Shoulder & 2.88 & 5.64 & 2.8 & 9.1 & 6.7 & 12.7 \\
\hline Elbow & 2.44 & - & - & 29.8 & 25.2 & 20.3 \\
\hline Hand & - & - & - & 151.5 & 181.7 & 14.5 \\
\hline Hip & 2.76 & 3.28 & 3.36 & 6.0 & 6.5 & 10.1 \\
\hline Knee & 1.31 & - & - & 8.8 & 16.8 & 35.1 \\
\hline Foot & - & - & - & 19.8 & 25.2 & 38.1 \\
\hline
\end{tabular}



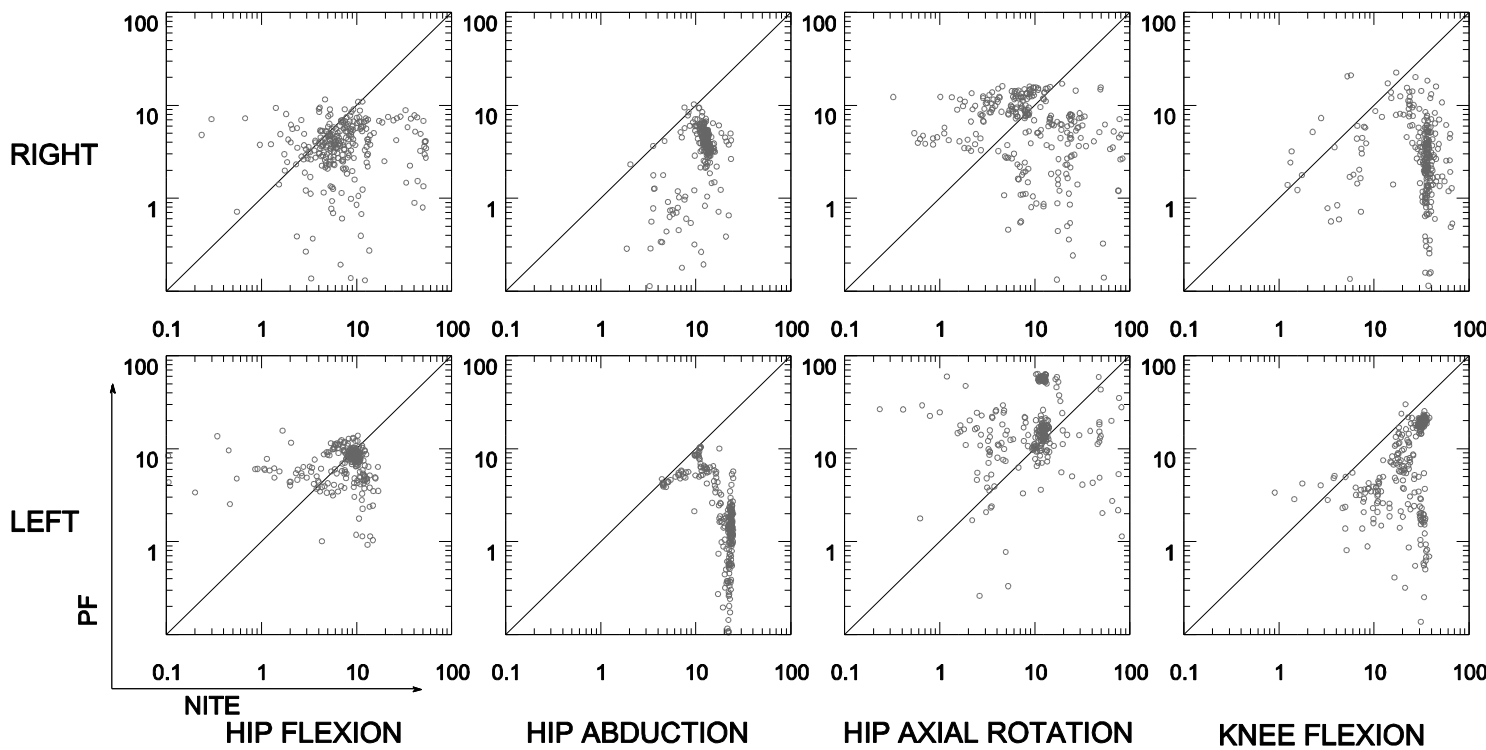

Figure 2 - NITE vs. particle filter (PF) errors in Euler angles of hips and knees for all the exercises. Absolute values in degrees (axes in logarithmic scales). Points in the right bottom sectors represent instants where NITE error was higher than PF error, and vice versa.

Considering the set of all measurements, the improvement provided by the PF to hips and knees $\left(\Delta \boldsymbol{\varepsilon}_{t}^{H}\right.$ and $\Delta \boldsymbol{\varepsilon}_{t}^{K}$, respectively) was clearly positive for the right-hand side, but small for the left-hand side (Table 2). Wilcoxon's test showed that the average of those variables was significantly positive $(W>10, P<.001)$ in all cases, except for the left hip, where the (also positive) difference was not significant ( $W=1.35, P=.176$ ).

When the individual exercises were considered, PF errors were often similar to NITE errors, although there are relatively more cases when NITE errors were greater and fewer cases when PF errors were greater. For side hip and tandem, the PF performed generally better, especially for the right-hand side. On the other hand, left hip axial rotation presented greater PF errors in all exercises. The values of $\Delta \boldsymbol{\varepsilon}_{t}^{H}$ and $\Delta \boldsymbol{\varepsilon}_{t}^{K}$ were small in most cases, but again, tended to be positive. Their interquartile ranges were fully positive in 8 cases, and only slightly negative in 1 out of 20 cases (left $\Delta \boldsymbol{\varepsilon}_{t}^{K}$ for the side hip exercise). 
Table 2. Summary of joint rotation errors associated with the NITE and particle filter algorithms. Mean values are given for each joint angle and the difference of attitude errors $\left(\Delta \boldsymbol{\varepsilon}_{t}^{H}, \Delta \boldsymbol{\varepsilon}_{t}^{K}\right)$, together with the interquartile ranges (25th and 75 th percentiles), given the strong non-normality of the distributions. All values in degrees.

\begin{tabular}{|c|c|c|c|c|c|c|c|c|c|c|}
\hline & \multicolumn{2}{|c|}{ Hip flex. } & \multicolumn{2}{|c|}{ Hip abd. } & \multicolumn{2}{|c|}{ Hip rot. } & \multirow[b]{2}{*}{$\Delta \boldsymbol{\varepsilon}_{t}^{H}$} & \multicolumn{2}{|c|}{ Knee flex. } & \multirow[b]{2}{*}{$\Delta \boldsymbol{\varepsilon}_{t}^{K}$} \\
\hline & NITE & $\mathrm{PF}$ & NITE & $\mathrm{PF}$ & NITE & $\mathrm{PF}$ & & NITE & $\mathrm{PF}$ & \\
\hline \multicolumn{11}{|l|}{ Right-hand side } \\
\hline \multicolumn{11}{|l|}{ Back knee } \\
\hline mean & 7.7 & 5.0 & 1.6 & 1.7 & 6.3 & 12.3 & -2.9 & 10.4 & 10.1 & 0.3 \\
\hline p25 & 4.6 & 2.8 & 0.4 & 0.6 & 3.0 & 9.7 & -8.6 & 6.3 & 1.7 & -9.5 \\
\hline p75 & 10.7 & 6.8 & 2.4 & 2.7 & 9.3 & 14.7 & 2.6 & 13.3 & 16.7 & 10.6 \\
\hline \multicolumn{11}{|l|}{ Sit + front knee } \\
\hline mean & 11.1 & 8.1 & 8.3 & 3.5 & 8.9 & 19.9 & -4.2 & 8.5 & 8.9 & -0.4 \\
\hline p25 & 3.1 & 3.0 & 6.4 & 2.1 & 2.7 & 18.1 & -10.3 & 5.5 & 4.3 & -5.6 \\
\hline p75 & 17.6 & 12.1 & 9.5 & 5.0 & 11.9 & 22.7 & 1.6 & 11.9 & 13.2 & 4.0 \\
\hline \multicolumn{11}{|l|}{ Side hip } \\
\hline mean & 46.1 & 6.8 & 36.9 & 2.2 & 28.2 & 14.7 & 56.5 & 37.3 & 2.8 & 34.5 \\
\hline p25 & 13.2 & 5.2 & 12.7 & 0.7 & 21.6 & 7.0 & 20.6 & 3.7 & 1.4 & 1.1 \\
\hline p75 & 55.8 & 8.2 & 65.9 & 2.9 & 32.0 & 20.0 & 110.5 & 30.5 & 4.0 & 26.6 \\
\hline \multicolumn{11}{|l|}{ Step } \\
\hline mean & 7.8 & 6.4 & 3.8 & 3.5 & 6.9 & 12.1 & -1.7 & 8.0 & 5.3 & 2.8 \\
\hline p25 & 2.9 & 4.7 & 1.1 & 2.1 & 2.8 & 8.0 & -4.7 & 5.7 & 3.4 & 0.2 \\
\hline p75 & 11.7 & 8.2 & 4.7 & 4.6 & 10.9 & 16.1 & 3.0 & 10.4 & 7.0 & 5.3 \\
\hline \multicolumn{11}{|l|}{ Tandem } \\
\hline mean & 9.2 & 4.2 & 12.6 & 4.3 & 12.5 & 5.9 & 14.4 & 31.8 & 4.0 & 27.9 \\
\hline p25 & 2.9 & 2.6 & 12.1 & 3.6 & 4.6 & 1.8 & 6.0 & 29.0 & 1.4 & 23.3 \\
\hline p75 & 10.6 & 5.6 & 13.9 & 5.2 & 13.3 & 10.6 & 18.9 & 37.0 & 4.9 & 34.6 \\
\hline \multicolumn{11}{|l|}{ All measures } \\
\hline mean & 16.6 & 6.2 & 13.0 & 3.2 & 12.8 & 12.7 & 13.1 & 19.3 & 5.6 & 13.7 \\
\hline p25 & 3.7 & 3.8 & 2.1 & 1.4 & 4.0 & 6.8 & -3.9 & 5.9 & 2.1 & -0.1 \\
\hline p75 & 14.4 & 8.1 & 13.5 & 4.6 & 18.0 & 17.8 & 18.3 & 17.7 & 7.2 & 21.8 \\
\hline \multicolumn{11}{|l|}{ Left-hand side } \\
\hline \multicolumn{11}{|l|}{ Back knee } \\
\hline mean & 2.8 & 6.0 & 20.9 & 4.1 & 5.2 & 14.9 & 4.2 & 6.9 & 3.8 & 3.1 \\
\hline p25 & 1.1 & 4.8 & 20.0 & 2.4 & 2.3 & 5.3 & -1.8 & 3.4 & 1.5 & -1.1 \\
\hline p75 & 4.3 & 7.2 & 21.5 & 5.2 & 8.1 & 22.6 & 11.4 & 9.5 & 5.5 & 6.6 \\
\hline \multicolumn{11}{|l|}{ Sit + front knee } \\
\hline mean & 13.5 & 6.7 & 7.3 & 3.4 & 5.3 & 6.6 & 6.4 & 5.8 & 6.8 & -1.0 \\
\hline p25 & 4.9 & 1.5 & 5.2 & 1.9 & 2.6 & 4.5 & 0.4 & 2.9 & 4.4 & -4.0 \\
\hline p75 & 17.8 & 10.0 & 9.4 & 4.6 & 6.3 & 8.6 & 9.3 & 6.4 & 8.2 & 1.9 \\
\hline \multicolumn{11}{|l|}{ Side hip } \\
\hline mean & 3.6 & 3.5 & 20.5 & 2.8 & 5.8 & 24.3 & -1.9 & 3.2 & 3.1 & 0.1 \\
\hline p25 & 0.5 & 1.8 & 21.1 & 1.5 & 3.9 & 11.3 & -12.8 & 1.2 & 1.0 & -2.5 \\
\hline p75 & 4.2 & 4.9 & 26.7 & 4.1 & 7.4 & 38.9 & 9.0 & 5.1 & 5.1 & 3.0 \\
\hline Step & & & & & & & & & & \\
\hline mean & 7.4 & 5.4 & 4.5 & 5.0 & 5.2 & 14.1 & -6.4 & 7.7 & 6.5 & 1.2 \\
\hline p25 & 6.2 & 2.8 & 3.0 & 3.1 & 2.2 & 6.3 & -9.5 & 7.0 & 5.1 & -1.5 \\
\hline $\mathrm{p} 75$ & 9.1 & 6.2 & 5.9 & 6.8 & 7.6 & 15.8 & 1.0 & 10.0 & 8.0 & 3.6 \\
\hline Tandem & & & & & & & & & & \\
\hline mean & 5.9 & 7.0 & 19.8 & 3.2 & 11.7 & 17.9 & 5.0 & 22.5 & 12.1 & 10.4 \\
\hline p25 & 4.3 & 3.9 & 15.7 & 0.8 & 5.8 & 10.0 & -1.0 & 12.8 & 4.1 & 6.8 \\
\hline p75 & 6.5 & 9.3 & 24.7 & 6.1 & 10.1 & 18.5 & 11.3 & 31.1 & 19.8 & 12.6 \\
\hline All measures & & & & & & & & & & \\
\hline mean & 6.9 & 5.6 & 13.0 & 3.8 & 6.7 & 15.8 & 0.1 & 9.4 & 6.7 & 2.7 \\
\hline p25 & 3.1 & 2.8 & 4.9 & 1.8 & 3.0 & 6.6 & -6.4 & 3.2 & 3.3 & -2.0 \\
\hline p75 & 8.9 & 7.3 & 23.2 & 5.3 & 8.5 & 19.5 & 8.4 & 10.8 & 8.1 & 5.7 \\
\hline
\end{tabular}



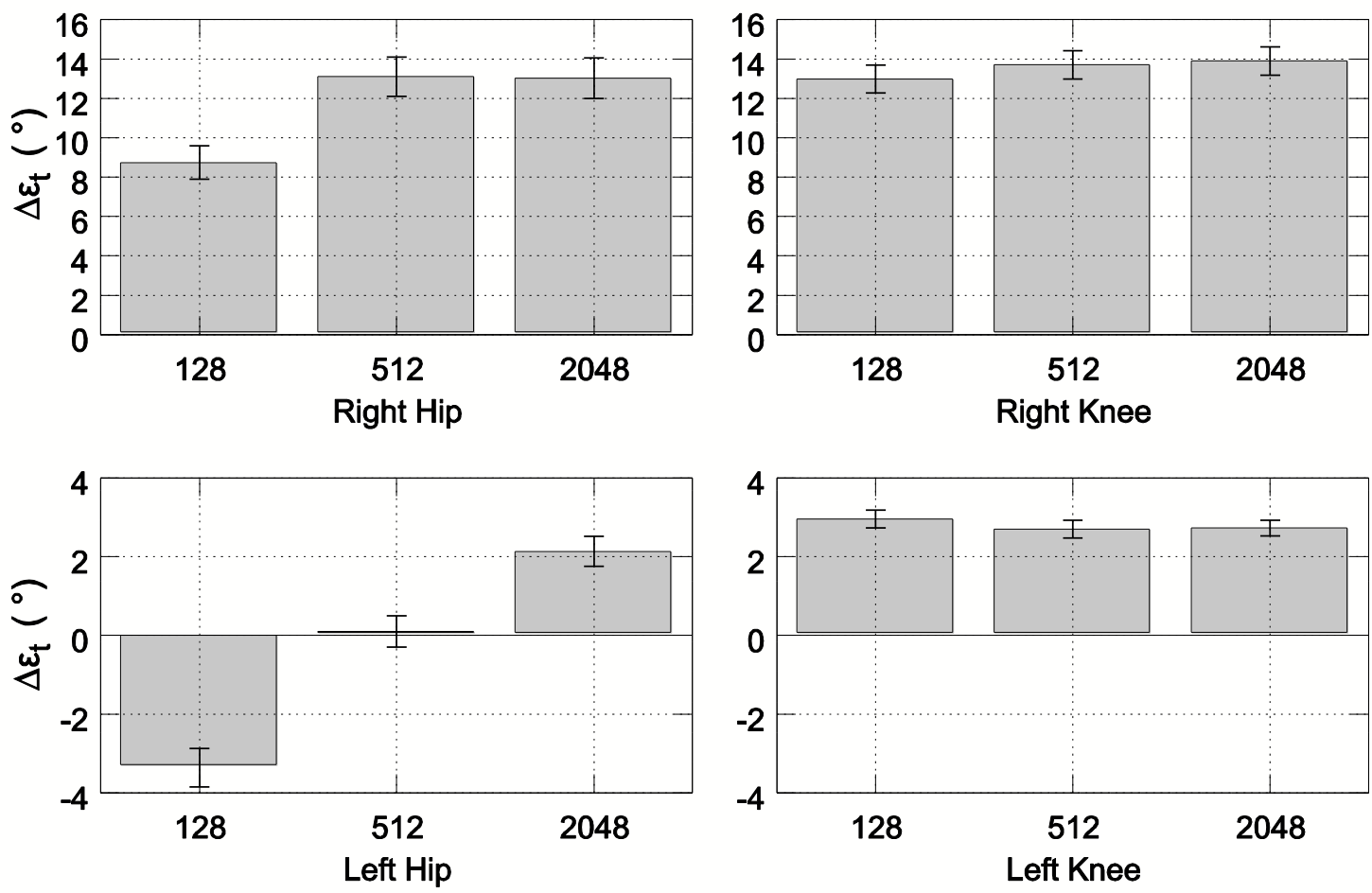

Figure 3- Mean and standard error of the difference between particle filter and NITE joint orientation errors $\left(\Delta \boldsymbol{\varepsilon}_{t}^{j}\right)$ for 128,512 , and 2048 particles.

Values in degrees.

The average improvement in hip rotations (but not of knee rotations) increased with the number of particles (Figure 3). However, even the gain in hips (around $6^{\circ}$ from 128 to 2048 particles) was small compared with the wide dispersion of errors that had been observed.

\section{Discussion}

This study presents an alternative to the methods used by Kinect middleware to calculate joint rotations. NITE errors often exceeded $10^{\circ}$ (even after correcting accidental "flips") except for hip flexion, unlike in previous studies. ${ }^{15}$ A possible explanation for this discrepancy is that the gold standard used by other authors was obtained by an inverse kinematic analysis of a simple skeleton model, formed by onedimensional lines linking joints. That model has the same problems of indeterminations and singularities reported for NITE algorithms, and could mask NITE errors. The gold standard of the present study was obtained from measurements with a greater number of markers and therefore, could be considered more valid. 
The error of the PF was typically below $10^{\circ}$, except for hip axial rotation. This was comparable with NITE errors and one order of magnitude lower for hip abduction/adduction and knee flexion. The rotations calculated by the PF were more reliable for joints in motion.

PF and related techniques are often employed for motion tracking, using data from inertial sensors or more complex optical data from marker-less motion capture. This study demonstrated that they can also be used with simpler, high-level data such as the position of a small number of anatomical landmarks. In addition to reducing errors, PF have the advantage of giving smooth angle trajectories, coherent with skeletons of fixed anthropometry. To obtain those properties with inverse kinematics, the analysis should be complicated with nonlinear optimization techniques. ${ }^{28}$

A limitation of this study is that it was conducted with just one subject in a laboratory. The intended application of this technology involves measuring many people in various home environments, such that the dispersions of $f\left(\boldsymbol{\theta} \mid \boldsymbol{\theta}_{t-1}\right)$ and $g\left(\mathbf{y} \mid \boldsymbol{\theta}_{t}\right)$ may be larger than the values observed in the experiment. However, the PF might still converge if there are a sufficient number of particles. ${ }^{29}$ The results show that hip errors are more sensitive to the number of particles, especially if the degree of motion is small. The required computational resources grow proportionally with the number of particles, but other mathematical approaches could be attempted to counteract that problem, such as Unscented Kalman Filters. ${ }^{30}$ Better performance could also be obtained by using a state space based on positions, instead of joint angles, whose effect on the observed posture is accumulated across the kinematic chains. ${ }^{31}$

This study was limited to the analysis of lower limb angles, as they were the ones of interest for the balance-assessment and training exercises considered in the present research framework. For less specific applications, the methodology could be easily applied to the analysis of upper limbs.

Funding: This work has been undertaken within the framework of the iStoppFalls project, which has received funding from the European Community (grant agreement FP7-ICT-2011-7-287361) and the Australian Government.

Conflict of interest disclosure: No conflict of interest exists for any of the authors of this paper. 


\section{References}

1. Di Tore S, D’Elia F, Aiello P, Carlomagno N, Sibilio M. Didactics, movement and technology: new frontiers of the human-machine interaction. J Hum Sport Exerc - University of Alicante. 2012;7(1Proc):S178-S184.

2. Pigford T, Andrews AW. Feasibility and Benefit of Using the Nintendo Wii Fit for Balance Rehabilitation in an Elderly Patient Experiencing Recurrent Falls. $J$ Student Phys Ther Res. 2010;2(1):12-20.

3. Young W, Ferguson S, Brault S, Craig C. Assessing and training standing balance in older adults: A novel approach using the "Nintendo Wii" Balance Board. Gait Posture. 2011;33(2):303-305.

4. Agmon M, Perry CK, Phelan E, Demiris G, Nguyen HQ. A pilot study of Wii Fit exergames to improve balance in older adults. $J$ Geriatr Phys Ther. 2011;34(4):161-167.

5. Garcia JA, Felix Navarro K, Schoene D, Smith ST, Pisan Y. Exergames for the elderly: towards an embedded Kinect-based clinical test of falls risk. Stud Health Technol Inform. 2012;178:51-57.

6. Lloréns R, Alcañiz M, Colomer C, Navarro MD. Balance recovery through virtual stepping exercises using Kinect skeleton tracking: a follow-up study with chronic stroke patients. Stud Health Technol Inform. 2012;181:108-112.

7. Smith ST, Schoene D. The use of exercise-based videogames for training and rehabilitation of physical function in older adults: current practice and guidelines for future research. Aging Health. 2012;8(3):243-252.

8. PrimeSense Inc. Prime Sensor ${ }^{\mathrm{TM}}$ NITE 1.3 Algorithms notes. 2010. http://pr.cs.cornell.edu/humanactivities/data/NITE.pdf. Accessed January 25, 2013.

9. Chang C-Y, Lange B, Zhang $\mathrm{M}$, et al. Towards pervasive physical rehabilitation using Microsoft Kinect. In: 2012 6th International Conference on Pervasive Computing Technologies for Healthcare (PervasiveHealth); 2012:159-162.

10. Schonauer C, Pintaric T, Kaufmann H, Jansen - Kosterink S, Vollenbroek-Hutten M. Chronic pain rehabilitation with a serious game using multimodal input. In: 2011 International Conference on Virtual Rehabilitation (ICVR); 2011:1-8.

11. Shotton J, Fitzgibbon A, Cook M, et al. Real-time human pose recognition in parts from single depth images. In: 2011 IEEE Conference on Computer Vision and Pattern Recognition (CVPR); 2011:1297-1304.

12. Clark RA, Pua Y-H, Fortin K, et al. Validity of the Microsoft Kinect for assessment of postural control. Gait Posture. 2012;36(3):372-377.

13. Obdrzalek S, Kurillo G, Ofli F, et al. Accuracy and robustness of Kinect pose estimation in the context of coaching of elderly population. In: 2012 Annual 
International Conference of the IEEE Engineering in Medicine and Biology Society (EMBC); 2012:1188-1193.

14. Samejima I, Maki K, Kagami S, Kouchi M, Mizoguchi H. A body dimensions estimation method of subject from a few measurement items using KINECT. In: 2012 IEEE International Conference on Systems, Man, and Cybernetics (SMC); 2012:3384-3389.

15. Fernández-Baena A, Susin A, Lligadas X. Biomechanical Validation of Upper-Body and Lower-Body Joint Movements of Kinect Motion Capture Data for Rehabilitation Treatments. In: 2012 4th International Conference on Intelligent Networking and Collaborative Systems (INCoS); 2012:656-661.

16. Wieching R, Kaartinen N, De Rosario H, et al. A New Approach for Personalised falls risk prediction and Prevention: Tailored Exercises. Unobtrusive Sensing \& Advanced Reasoning. J Aging Phys Act. 2012;20(S):S120-S124.

17. Wu G, Siegler S, Allard P, et al. ISB recommendation on definitions of joint coordinate system of various joints for the reporting of human joint motionpart I: ankle, hip, and spine. J Biomech. 2002;35(4):543-548.

18. Wu G, Van der Helm FCT, (DirkJan) Veeger HEJ, et al. ISB recommendation on definitions of joint coordinate systems of various joints for the reporting of human joint motion-Part II: shoulder, elbow, wrist and hand. $J$ Biomech. 2005;38(5):981-992.

19. Woltring HJ. 3-D attitude representation of human joints: A standardization proposal. J Biomech. 1994;27(12):1399-1414.

20. Cappe O, Godsill SJ, Moulines E. An Overview of Existing Methods and Recent Advances in Sequential Monte Carlo. Proc IEEE. 2007;95(5):899-924.

21. Anon. Range of Motion Evaluation Chart. Washington State Department of Social \& Health Services; 2003. http://www.dshs.wa.gov/pdf/ms/forms/13_585a.pdf. Accessed January 25, 2013.

22. Soucie JM, Wang C, Forsyth A, et al. Range of motion measurements: reference values and a database for comparison studies. Haemophilia. 2011;17(3):500507.

23. Page A, De Rosario H, Mata V, Atienza C. Experimental Analysis of Rigid Body Motion. A Vector Method to Determine Finite and Infinitesimal Displacements From Point Coordinates. J Mech Des. 2009;131(3):031005.

24. Robertson MC, Campbell AJ. Otago Exercise Programme to Prevent Falls in Older Adults. Wellington: Accident Compensation Corporation; 2003.

25. Tiedemann A, Lord SR, Sherrington C. The Development and Validation of a Brief Performance-Based Fall Risk Assessment Tool for Use in Primary Care. $J$ Gerontol A Biol Sci Med Sci. 2010;65A(8):896-903. 
26. De Rosario H, Page A, Besa A, Mata V, Conejero E. Kinematic description of soft tissue artifacts: quantifying rigid versus deformation components and their relation with bone motion. Med Biol Eng Comput. 2012;50(11):1173-1181.

27. Eaton JW, Bateman D, Hauberg S. GNU Octave Manual Version 3. Godalming, UK: Network Theory Limited; 2008.

28. Schwarz LA, Mkhitaryan A, Mateus D, Navab N. Human skeleton tracking from depth data using geodesic distances and optical flow. Image Vis Comput. 2012;30(3):217-226.

29. 1. Crisan D, Doucet A. A survey of convergence results on particle filtering methods for practitioners. IEEE Trans Signal Process. 2002;50(3):736-746. doi:10.1109/78.984773.

30. Larsen ABL, Hauberg S, Pedersen KS. Unscented Kalman Filtering for Articulated Human Tracking. In: Heyden A, Kahl F, eds. Image Analysis. Lecture Notes in Computer Science. Springer Berlin Heidelberg; 2011:228-237.

31. Hauberg S, Sommer S, Pedersen KS. Natural metrics and least-committed priors for articulated tracking. Image Vis Comput. 2012;30(6-7):453-461. 